\title{
Prévalence de la consommation de produits modifiant la vigilance chez des transporteurs routiers dans la Région Nord-Pas-de-Calais
}

\author{
Use of compounds altering vigilance \\ performance : results of prevalence in \\ haulage drivers in the Nord-Pas-de-Calais \\ Region
}

\section{Laurence LABAT $^{(1) *}$, Betty DEHON ${ }^{(1)}$, Michel LHERMITTE ${ }^{(1)}$, et le groupe régional "Toxicomanie et Travail"(2)}

(1) Service de Toxicologie et Génopathies, CHRU de Lille, Boulevard du Professeur Jules Leclercq 59037 LILLE CEDEX (2) Institut de Santé au Travail du Nord de la France, 1, Avenue Oscar Lambert - 59037 LILLE CEDEX

*Auteur à qui adresser la correspondance : Laurence LABAT, Service de Toxicologie et Génopathies, CHRU de Lille, Boulevard du Professeur Jules Leclercq - 59037 LILLE CEDEX

Ce travail a été présenté en partie au XII ${ }^{\mathrm{eme}}$ Congrès annuel de la SFTA à Porticcio, mai 2004

(Reçu le 3 décembre 2004 ; accepté après modifications le 30 décembre 2004)

\section{$R E ́ S U M E ́$}

En 1995, une enquête avait montré que le nombre de consommateurs de substances modifiant la vigilance était significativement plus important chez les salariés occupant un poste de sûreté/sécurité que dans le reste de la population active. Pour vérifier si cette tendance se poursuit, une nou. velle étude multicentrique pour la branche professionnelle des transporteurs routiers a été réalisée en 2003-2004 dans la Région Nord-Pas-de-Calais. Cette étude avait pour but de permettre une harmonisation des pratiques professionnelles des médecins du travail en proposant une politique de prévention et de dépistage, en validant les techniques à utiliser et la conduite à tenir en cas de dépistage positif.

\section{SUMMARY}

A previous study conducted in 1995 showed that psychoactive drugs intake was higher in safety/security workstations than in the rest of the labour force. In order to verify if this tendency is always the same, we conducted a new study in 2003-2004 in the Nord-Pas-de-Calais region, restricted to haulage drivers. The aim of this study was to allow to harmonize the professional practice of the occupational physicians, proposing drug prevention and drug testing policies, validating the analytical methods and the guidelines in case of positive testing results.

One thousand haulage drivers were studied. Urine were tested for amphetamines, cannabis, cocaine, opiates, benzodia- 
Dans cette étude, mille chauffeurs routiers sont inclus. On réalise un dépistage urinaire par des tests immunologiques de la méthadone, des benzodiazépines, de la cocaïne, des amphétamines, des opiacés, des cannabinoïdes et de la buprénorphine. L'éthanol est dosé dans les urines par mêthode enzymatique. Tous les dépistages positifs sont confirmés par une technique de chromatographie couplée à la spectrométrie de masse. Les résultats exprimés en pourcentage de positifs sont les suivants: opiacés $(4,1 \%)$, cannabinoïdes $(8,5 \%)$, cocaïne $(0,1 \%)$, amphétamines $(0,3 \%)$, buprénorphine $(1,8 \%)$, méthadone $(0,5 \%)$, benzodiazépines $(0,4 \%)$ et alcool $(5 \%)$.

Seul dans un cas, la 6-monoacétylmorphine a été identifiée dans des urines positives en opiacés. Les autres opiacés étaient pour la majorité des antitussifs. Le faible nombre de résultats positifs pour les benzodiazépines dans cette étude peut s'expliquer par la sensibilité et la spécificité du test rapide utilisé. Ces résultats confirment ceux de l'étude de 1995 pour la consommation de cannabis et d'alcool chez des travailleurs affectés à des postes de sûreté/sécurité. Les pourcentages de dépistages positifs aux traitements de substitution (méthadone et buprénorphine) sont élevés, huit fois plus que dans la population active. Au regard de ces résultats, il semble intéressant de pouvoir étendre l'étude à la consommation des autres classes de médicaments psychoactifs, en utilisant une méthode de screening plus large comme la chromatographie liquide haute performance couplée à la spectrométrie de masse.

\section{MOTS-CLÉS}

Poste de sûreté/sécurité, Psychotropes.

\section{Introduction}

Au cours des visites médicales prévues par la législation de la santé au travail, on peut effectuer une recherche urinaire de stupéfiants ou de médicaments après information et acceptation préalable du salarié. Les résultats sont soumis au respect du guide de bonne conduite en vigueur, et sont couverts de façon absolue par le secret médical. Pour les postes de sûreté/sécurité (PSS), le médecin effectuant la visite émet un avis sur l'aptitude de l'intéressé à occuper son poste.

En 1995, une enquête avait montré que le nombre de consommateurs de substances altérant la vigilance était significativement plus important chez les salariés occupant un PSS que dans le reste de la population active $(1,2)$. Par ailleurs, même si le nombre d'accidents impliquant un poids lourd diminue régulièrement depuis 1985, l'indice de gravité lui est en augmentation. C'est dans ce contexte que le groupe "Toxicomanie et Travail" de la Région Nord-Pas-deCalais a mis en place une étude multicentrique pour la branche professionnelle des transporteurs routiers des secteurs de Douai, Hénin-Beaumont et de Lesquin. Cette étude a pour objectif d'harmoniser les pratiques professionnelles des médecins du travail ayant en char- zepines, buprenorphine and methadone by immunoanalysis. Urine ethanol determinations were performed by $A D H$ method. Positive urines were then tested by gas chromatography or liquid chromatography coupled to mass spectrometry. Out of the 1000 drivers, cannabinoids were detected in 85 cases, opiates in 41 cases, amphetamines in 3 cases and cocaine in only I case. Buprenorphine was detected in 18 cases, methadone in 5 cases and benzodiazepines in 4 cases. Urine ethanol was positive in 50 cases. We found only one case with 6-monoacetylmorphine. Other positive opiates were determined as cough mixtures. The few cases of benzodiazepines positive urines could be explained by the sensitivity and the specificity of the test we used.

All these results strength those of the previous study for cannabis and ethanol in safety/security workstations. Positive results for methadone and buprenorphine are 8 times higher than in the general population. In conclusion, the authors think that it will be of a great interest to test urine of drivers for other classes of psychoactive drugs, using a liquid chromatography-mass spectrometry method.

\section{KEY-WORDS}

safety/security workstation, Psychotropes.

ge des entreprises de transports routiers, en proposant une politique commune de prévention et de dépistage, en validant les techniques à utiliser et la conduite à tenir en cas de dépistage positif.

\section{Matériels et méthodes}

\section{Population étudiée et recueil des échan- tillons :}

Le protocole de cette nouvelle étude a été approuvé par le Conseil National de l'Ordre des Médecins. Il est conforme aux exigences éthiques et scientifiques respectant notamment l'anonymat des salariés qui sont préalablement informés de l'étude et rentre dans le cadre des préoccupations actuelles nationales sur la sécurité routière.

Mille chauffeurs routiers ont été inclus dans l'étude, le seul critère de sélection étant leur emploi. Pour le recrutement, quatre sites dans la Région Nord-Pas-deCalais ont été choisis : le Centre Régional des Transports de Lesquin (Nord), deux entreprises dans le secteur de Douai (Nord) et une entreprise à HéninBeaumont (Pas-de-Calais). Afin de les distinguer, un des centre de Douai, centre de formation pour chauf- 
feurs routiers sera nommé Douai 2. Les recueils des données et des prélèvements urinaires ont été réalisés, au cours de la visite annuelle, de la visite d'embauche ou de la visite de reprise, à l'exclusion de tout autre examen, en collaboration avec les médecins des services de santé au travail. Les échantillons d'urines ont été collectés de façon anonyme. Les seuls renseignements étaient le zonage de l'entreprise, l'âge, le sexe, le type de la visite et le jour de prélèvement.

Les urines ont été collectées dans des pots en polypropylène. Elles sont émises sur place dans l'entreprise dans une pièce dépourvue de point d'eau et de produits d'entretien pour éviter toute falsification. Le médecin du travail a vérifié l'absence de nitrites, la température (entre 32,5 et $37,5^{\circ} \mathrm{C}$ ), la densité (entre 1,007 et 1,035 ), le $\mathrm{pH}$ (entre 4,8 et 8 ) des urines. Les échantillons urinaires sont placés à $4^{\circ} \mathrm{C}$ immédiatement puis congelés le soir de la vacation. Ils sont acheminés congelés au laboratoire.

\section{Méthodes}

On réalise un dépistage urinaire (test Triage $^{\mathrm{TM}}$, Biosite, San Diego, USA) de la méthadone (seuil de détection $=300 \mathrm{ng} / \mathrm{mL}$ ), des benzodiazépines (300 $\mathrm{ng} / \mathrm{mL})$, de la cocaine $(300 \mathrm{ng} / \mathrm{mL})$, des amphétamines $(1000 \mathrm{ng} / \mathrm{mL})$, des opiacés (300 $\mathrm{ng} / \mathrm{mL}$ ) et des cannabinoïdes $(50 \mathrm{ng} / \mathrm{mL})$. La recherche de la buprénorphine (1 $\mathrm{ng} / \mathrm{mL}$ ) est réalisée par une méthode Elisa (Microgenics, Fremont, USA). Tous les résultats positifs sont confirmés en chromatographie en phase gaz couplée à la spectrométrie de masse (CPG-SM) ou en chromatographie liquide couplée à la spectrométrie de masse (CLHP-SM) selon les méthodes déjà décrites dans le littérature (3 - 7). Pour l'identification des opiacés en CPG-SM, les urines ont été hydrolysées (8). Le dosage d'alcool est réalisé par méthode enzymatique (Dade Behring, Milton Keynes, UK) (seuil de détection $=0,1 \mathrm{~g} / \mathrm{L}$ ).

\section{Appareillage}

Les confirmations du dépistage de la cocaïne, des amphétamines, des opiacés, des cannabinoïdes ont été réalisées sur une CPG de type 6890 couplée à un spectromètre de masse de type 5973 (Agilent, Massy, France). Un passeur automatique de type 7683 permet l'injection des échantillons et l'ensemble des données est traité par le logiciel HPchem (Agilent).

Pour la méthadone, les benzodiazépines et la buprénorphine, les dépistages positifs ont été confirmés en CLHP-SM sur un appareil de type 2695 couplée à un spectromètre de masse de type Micromass ZQ (Waters, Saint Quentin en Yvelines, France). Les données sont acquises et traitées par un logiciel MassLynx version 4.0 (Waters).

\section{Résultats}

L'étude a débuté en juin 2003 . Les 1000 prélèvements ont fini d'être collectés et analysés en juillet 2004 . Les hommes représentent la majorité des salariés avec $99,20 \%$. L'âge moyen est de $36,7+/-9,8$ ans. La figure 1 représente la répartition de cette population de chauffeurs routiers en fonction du jour de la visite, de la classe d'âge et du type de visite. C'est une population assez jeune car près de $80 \%$ de salariés sont âgés de moins de 45 ans.
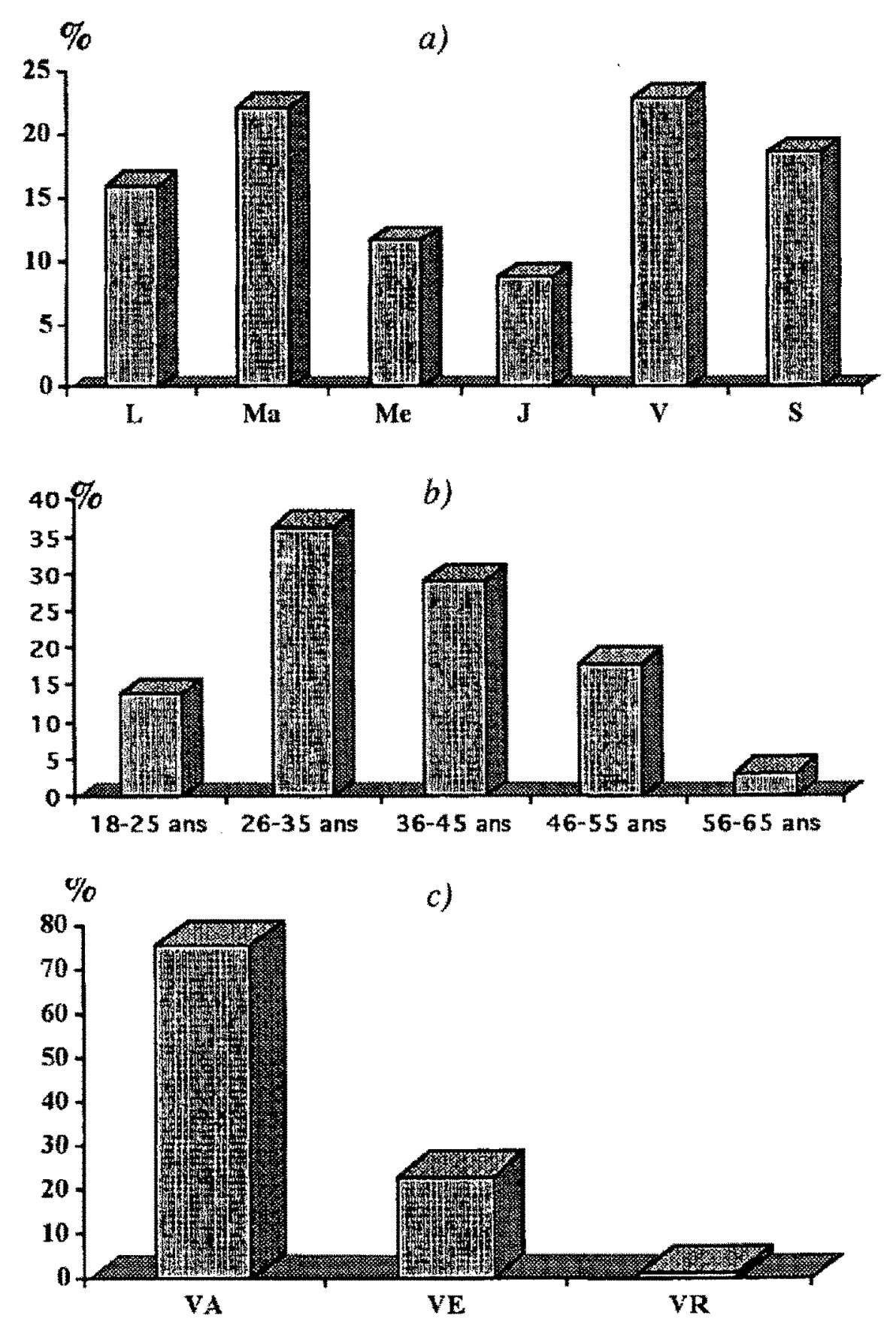

Figure I : Caractéristiques démographiques de la population étudiée: a) jour de visite ; b) classe d'âge ; c) type de visite avec $V A=$ visite annuelle, $V E=$ visite d'embauche et $V R=$ visite de reprise. 
Les prévalences des différentes substances recherchées dans cette étude, exprimées en pourcentage de positifs sont résumées dans le tableau I. On distingue les pourcentages de positifs dépistés par immunoanalyse ou méthode enzymatique et ceux confirmés par CPG-SM ou CLHP-SM.

Les 41 cas positifs en opiacés ont été identifiés en CPG-SM. Les résultats des identifications sont présentés dans le tableau II.

Tableau I : Prévalence des différentes substances recherchées sur la population étudiée (1000 individus).

\begin{tabular}{|l|c|c|}
\hline & $\begin{array}{c}\text { \% de dépistages positifs } \\
\text { par méthodes } \\
\text { immunologique ou } \\
\text { enzymatique }\end{array}$ & $\begin{array}{c}\text { \% de dépistages positifs } \\
\text { confirmés par CPG-SM } \\
\text { ou CLHP-SM }\end{array}$ \\
\hline Opiacés & 4,1 & 4,1 \\
Cannabis & 8,6 & 8,5 \\
Cocaïne & 0,1 & 0,1 \\
Amphétamines & 0,4 & 0,3 \\
Buprénorphine & 2,2 & 1,8 \\
Méthadone & 0,5 & 0,5 \\
Benzodiazépines & 0,4 & 0,4 \\
Éthanol & 5,0 & - \\
\hline
\end{tabular}

Tableau II : Identification des opiacés par CPG-SM.

\begin{tabular}{|l|c|}
\hline \multicolumn{1}{|c|}{ Identification des opiacés } & $\begin{array}{c}\text { Nombre de cas } \\
(\mathbf{n}=\mathbf{4 1})\end{array}$ \\
\hline 6-MAM & 1 \\
morphine & 4 \\
codéine & 6 \\
pholcodine & 23 \\
codéine + morphine & 6 \\
codéthyline + morphine + codéine & 1 \\
\hline
\end{tabular}

Cinq pour cent des urines contenaient de l'éthanol avec des concentrations comprises entre 0,1 et $8,72 \mathrm{~g} / \mathrm{L}$. L'âge moyen des salariés concemés est de $41,1+/-8,5$ ans. Leur répartition par classe d'âge est représenté dans la figure 2 .

Plus de huit pour cent d'urines ont été dépistées et confirmées positives en cannabis. A une exception près, toutes les urines des travailleurs dépistés cannabinoïdes positifs par la méthode immunologique ont été confirmées et seul le THC-COOH a été identifié. L'âge moyen des salariés concernés est de $28,4+1-6,1$ ans. La figure 2 illustre la répartition par classe d'âge des travailleurs dépistés positifs en cannabis. Cette popula- tion est plus jeune que la population dépistée positive en éthanol, avec notamment $22,6 \%$ de positifs chez les salariés âgés entre 18 et 25 ans. Pour l'ensemble des quatre centres, l'étude de la répartition de cette population de salariés en fonction du type de visite montre un pourcentage élevé de $14,7 \%$ pour les visites d'embauche.

La même étude réalisée en fonction du centre et du type de visite montre un résultat proche de $30 \%$ d'urines dépistées positives en cannabis sur le centre de Douai 2 dans le cas des visites annuelles. Les résultats sont présentés dans la figure 3 .

Plus de deux pour cent des salariés ont été dépistés positifs à un traitement de substitution aux opiacés : $1,8 \%$ des urines ont été dépistées et confirmées en buprénorphine et $0,8 \%$ en méthadone. Un salarié de l'étude consommait les deux médicaments. L'âge moyen des salariés sous traitement de substitution est de 32 ans pour la buprénorphine et de 26 ans pour la méthadone. La répartition de cette population en fonction des zones est représentée dans la figure 4. Les prévalences les plus élevées sont celles du centre Douai 2.

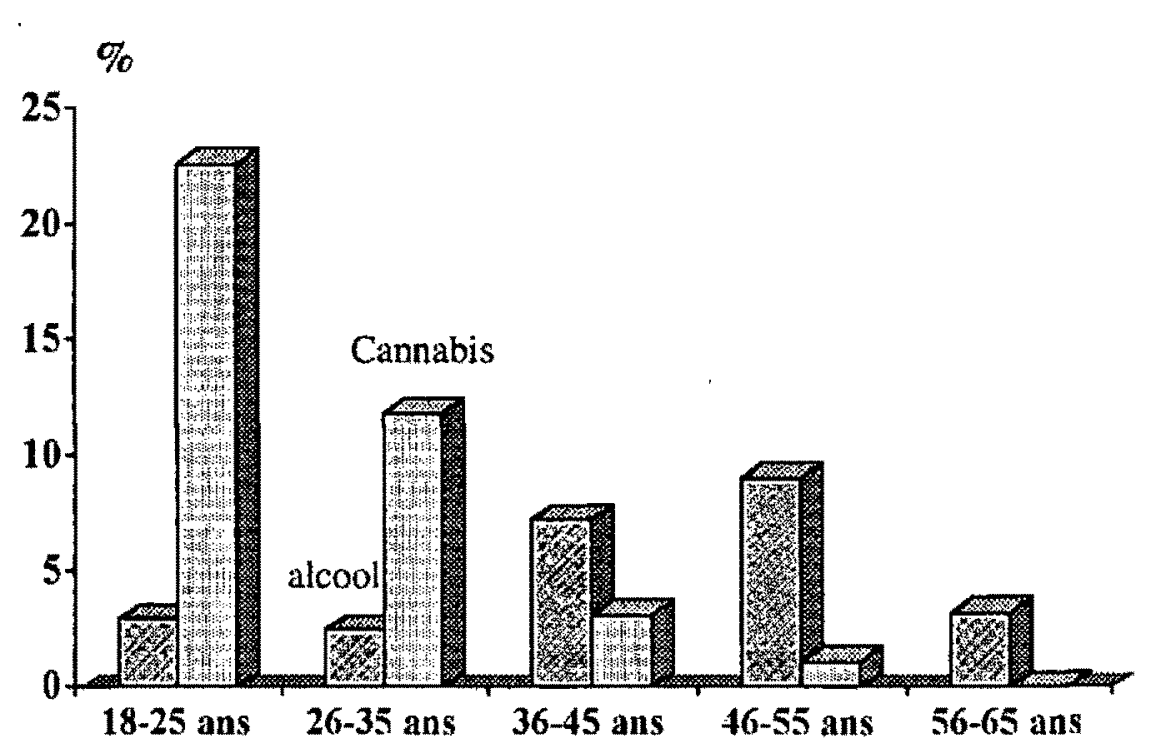

Figure 2 : Prévalence de l'alcool et du cannabis par classe d'âge.

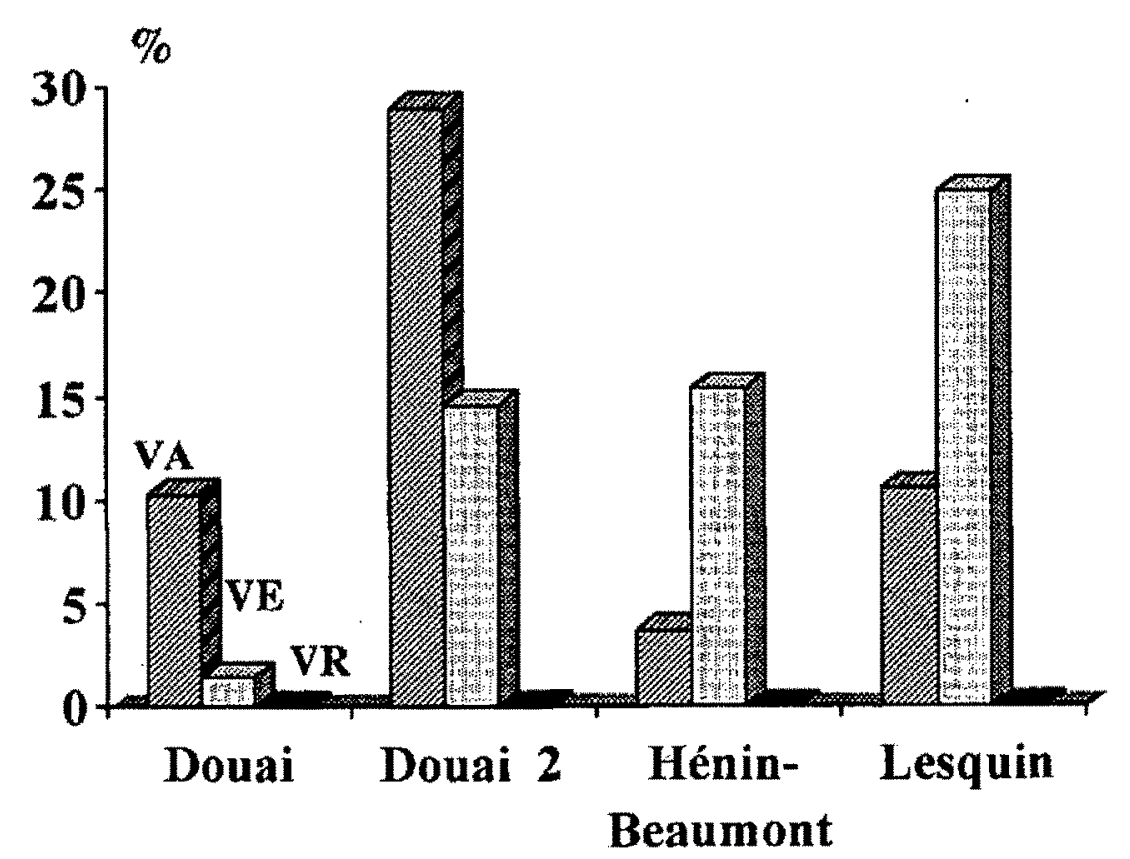

Figure 3 : Prévalence du cannabis dans les quatre centres étudiés en fonction du type de visite avec $V A=v i s i t e$ annuelle, $V E=$ visite d'embauche et $V R=$ visite de reprise. 


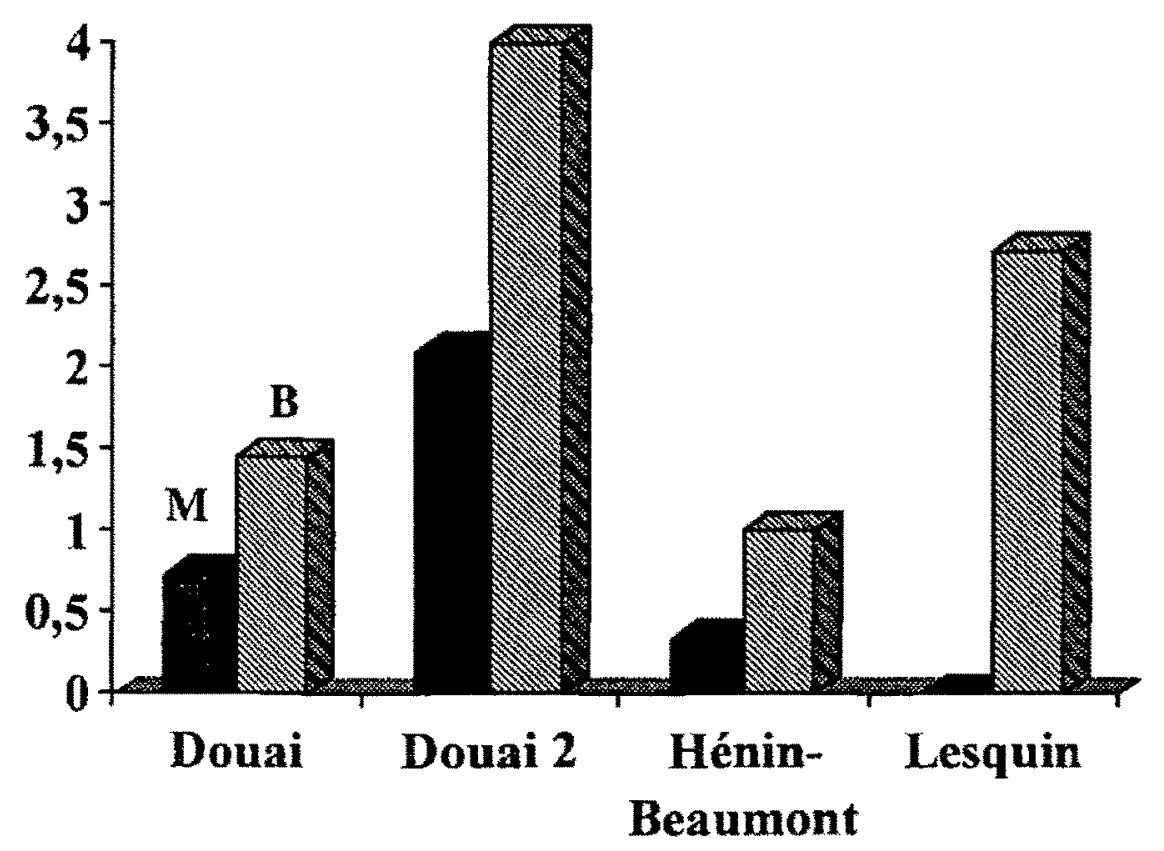

Figure 4 : Prévalence des traitements de substitution aux opiacés dans les quatre centres avec $M=$ méthadone, $B=$ buprénorphine.

\section{Discussion et Conclusion}

Peu de travaux ont été publiés sur la prévalence de la consommation de substances psychoactives pour des salariés occupant des postes de sûretélsécurité en France $(1,2)$. Seules deux études concernant les accidents de la circulation dans la Région Nord-Pas-deCalais avaient fait état des chauffeurs routiers $(9,10)$. Dans notre étude, l'inclusion d'une population de 1000 chauffeurs routiers apparaît suffisante pour rendre compte de l'état actuel de la situation dans quatre centres de la Région Nord-Pas-de-Calais. Les salariés et les employeurs ont été informés de l'étude mais le recueil des urines a été réalisé de façon anonyme, permettant d'évaluer de façon plus exacte la prévalence de la consommation aux substances pouvant modifier la vigilance.

Le dépistage des substances psychoactives dans les urines a été réalisé par un test rapide immunologique facilement utilisable par tout médecin du travail lors d'une consultation en entreprise. Il présente les avantages d'être rapide mais tout résultat positif pouvant servir au diagnostic médical d'une éventuelle toxicomanie doit être confirmé par une méthode de référence $(8,11)$. Ainsi, dans notre étude tous les dépistages positifs ont été vérifiés par CPG-SM ou CLHP-SM, ce qui différencie cette nouvelle étude de celle d'Haguenoer et coll. en 1997 (1).

En ce qui concerne le dépistage des opiacés, seule la technique en CPG-SM permet de conclure sur la nature des produits consommés (8). A une exception près d'un salarié identifié comme consommateur d'héroïne, seuls des opiacés licites ont été identifiés. Il s'agit essentiellement d'antitussifs et de leurs métabolites.
Comme il avait déjà été constaté dans la précédente étude (1), il est étonnant que le nombre de salariés ayant de l'alcool dans les urines soit inférieur à celui des salariés positifs en cannabis dans une région où l'alcoolisme est très fréquent. Il est cependant impossible de conclure sur ces résultats en se basant uniquement sur des concentrations d'éthanol dans les urines.

Le profil de consommation de l'alcool et du cannabis correspond assez bien avec les profils classiquement observés en fonction des classes d'âge : augmentation de l'alcool avec l'âge tandis qu'à l'inverse le cannabis est retrouvé chez les 18-25 ans en majorité et pas du tout chez les plus de 55 ans. La prévalence de la consommation de cannabis mesurée dans cette nouvelle étude est comparable à celle qui avait été obtenue pour des salariés occupant un PSS dans l'étude de Haguenoer et coll. (1). Dans les deux études, les tests immunologiques utilisés ont les mêmes seuils de positivité à $50 \mathrm{ng} / \mathrm{mL}$. En choisissant un seuil plus bas à 20 ou $25 \mathrm{ng} / \mathrm{mL}$ pour le dépistage urinaire du cannabis, on peut penser que cela permettrait d'augmenter certainement le nombre de sujets positifs.

Le faible pourcentage de résultats positifs pour les benzodiazépines dans cette étude peut s'expliquer par le manque de sensibilité et de spécificité du test immunologique utilisé, le test TRIAGE® ayant un seuil de positivité de $300 \mathrm{ng} / \mathrm{mL}$ en équivalent nordiazépam.

A la différence de la précédente étude réalisée dans le Nord-Pas-de-Calais (1), nous avons recherché l'éventuelle prise d'un traitement de substitution aux opiacés (méthadone et buprénorphine). Les résultats montrent que $2,2 \%$ des salariés testés avait consommé un traitement de substitution. D'après les sources de l'INSEE, 50000 personnes salariés en France sont sous traitement de substitution, soit $0,29 \%$ de la population active (12). On peut noter une prévalence presque 8 fois supérieure. Devant ces résultats, on peut se poser la question de l'orientation des personnes consommant de façon régulière des psychotropes vers des postes de sûreté/sécurité.

Seules les urines de 18 salariés sur les 22 dépistées positives en buprénorphine par la technique Elisa ont été confirmées en CLHP-SM (tableau I). Pour les quatre urines non confirmées, la buprénorphine et la norbuprénorphine n'ont pas été identifiées en spectrométrie de masse. Les quatre faux positifs s'expliquent certainement par le choix du seuil de positivité de la buprénorphine dans les urines à $1 \mathrm{ng} / \mathrm{mL}$ en méthode immunologique.

D'autre part, dans le cas d'une recherche de prise de traitement de substitution, le médecin du travail vérifie l'absence d'autres prises de psychotropes. Il s'inquiétera à l'interrogatoire d'une éventuelle prise de certaines 
benzodiazépines et médicaments apparentés comme le flunitrazépam, la zopiclone et le zolpidem, car aucune des ces molécules ne peut être dépistée par un test rapide immunologique comme le test TRIAGE utilisé dans notre étude. On peut donc s'interroger de l'intérêt de réaliser la recherche des benzodiazépines par un test rapide lors de la visite médicale du salarié. Seule une technique chromatographique performante comme la CLHP ou la CG couplées à la spectrométrie de masse permettrait de répondre aux questions que se posent le médecin du travail dans cette situation (6).

Enfin, en se basant uniquement sur des résultats de dépistages dans les urines, il est impossible d'affirmer qu'un salarié est un consommateur régulier d'une ou plusieurs substances psychoactives ou qu'il n'est pas rentré dans une démarche de soins. En fonction du contexte, les examens urinaires pourraient être complétés par des dosages sanguins ou l'analyse des cheveux, chaque milieu apportant des informations complémentaires.

Les prévalences mesurées dans notre étude confirment celles de l'étude de 1995 (1) pour la consommation de cannabis et d'alcool chez des travailleurs affectés à des postes de sûreté/sécurité. Les dépistages positifs sont en baisse pour les amphétamines, les opiacés et les benzodiazépinies. Les autres molécules n'avaient pas été recherchées dans la précédente étude. Au regard de ces premiers résultats, il semble intéressant d'envisager l'étude sur la consommation des autres classes de médicaments psychoactifs par une méthode de screening plus large comme la CLHP couplée à la barrette de diodes, voire la CLHP-SM.

\section{Remerciements}

Cette étude n'aurait pu être réalisée sans la participation active du groupe «Toxicomanie et travail» composée de J.C. Archange, médecin du travail SNCF Lille, J.M. Brillet et A. Chatfield, médecins inspecteurs régional du travail DRTEFP Lille, C. Delzenne, médecin du travail SMSED Douai, A. Doublet, médecin du travail AIMT Hénin-Beaumont, B. Fontaine, médecin du travail AMEST Lille, P. Frimat, Professeur de médecine du travail, CHRU de Lille, M.C. Marek, médecin du travail AIMT Hénin-beaumont, B. Pamart, médecin du travail Renault Douai, P. Pradeau, médecin du travail Cogéma La Hague, D. Tellier, médecin du travail AIMST Lille, M. Tonneau, chargée de projet ISTNF, A. Trichard, médecin du travail, CHRU de Lille, D. Vanberkel, secrétaire générale ISTNF Lille, $\mathrm{S}$. Vandenbussche, chargé de mission ISTNF.

Cette étude a été financée par un Programme Régional de Santé : PRS CCAR 2002-447 et par l'Institut de Santé au travail du Nord de la France (ISTNF).

\section{Références}

1. Haguenoer J.M., Hannothiaux M.H., Lahaye-Roussel M.C., Fontaine B., Legrand P.M., Shirali P., Pamart B., Brillet J.M., Btouck N., Bailly I., Bailly C., Frimat P. Prévalence des conduites toxicophiles en milieu professionnel : une étude dans la région du Nord Pas de Calais. Bulletin du Conseil Départemental du Nord, Ordre des Médecins, $1997 ; 80: 11-5$.

2. Fontaine B., Groupe Régional Toxicomanie et Travail. Substances psychoactives et travail. Approche épidémiologique. Ann. Toxicol. Anal. 2002 ; XIV (1) : 10-4.

3. Kintz P., Cirimele V., Pépin G., Marquet P., Deveaux M., Mura P. Identification et dosage des cannabinoides dans le sang total. Toxicorama, 1996 ; VII (2) : 29-33.

4. Marquet P., Lachatre G., Kintz P., Pépin G., Deveaux M., Mura P. Identification et dosage des principales drogues amphétaminiques dans le sang total par CPG-SM. Toxicorama, 1996 ; VII (2) : 23-8.

5. Gaillard Y., Pépin G., Marquet P., Kintz P., Deveaux M., Mura P. Identification et dosage de la benzoylecgonine, cocaïne, morphine et 6-acétylmorphine dans le sang. Toxicorama, 1996 ; VII (2): 17-22.

6. Tracqui A., Kintz P., Mangin P. HPLC/MS determination of buprenorphine and norbuprenorphine in biological fluids and hair samples. J. Forensic Sci. 1997 ; 42 (1) : 111-4.

7. Humbert L., Grisel F., Bondoux G., Lhermitte M. HPLC/Photodiode array detection combined with ESI/MS detection : a powerful tool for large screening of bioactive molecules in complex biological matrices. Elaboration of an UV/ESI/MS spectra library enabling fast and reliable compound identification. Ann. Toxicol. Anal. 2002 ; XIV (3) : 248.

8. Goullé J.P., Lacroix C. L'addiction en milieu professionnel : quelles techniques de confirmation après immunoanalyse ? Ann. Toxicol. Anal. 2002 ; XIV (1) : 27-32.

9. Deveaux M., Marson J.C., Goldstein P., Lhermitte M., Muller P. Alcool et médicaments psychotropes dans les accidents mortels de la circulation. Etude de 132 cas dans la région Nord-Pas-de-Calais. Sem. Hôp. Paris 1991 ; $67: 1372-6$.

10. Deveaux M., Marson J.C., Goldstein P., Lhermitte M., Muller P. Alcool et psychotropes chez les accidentés de la route dans la région Nord-Pas-de-Calais. Urgences $1989 ; 8: 123-7$.

11. Capolaghi B. Dépistage urinaire : les limites de l'immunoanalyse. Ann. Toxicol. Anal. 2002 ; XIV (1) : 24-6.

12. Cadet-Taïrou A., Costes J.M., Bello P.Y., Palle C. Les traitements de substitution en France : résultats récents 2004. Quel est le nombre d'usagers d'opiacés sous BHD ? Tendances. $2004 ; 37: 1-2$. 